\title{
A modeling study of asymmetries in plasma irregularity characteristics near gradient reversals
}

\author{
Leslie J. Lamarche and Roman A. Makarevich \\ Geophysical Institute and Department of Physics, University of Alaska Fairbanks, Fairbanks, Alaska, USA
}

Correspondence to: Leslie J. Lamarche (ljlamarche@alaska.edu)

Received: 24 March 2016 - Revised: 13 June 2016 - Accepted: 2 August 2016 - Published: 31 August 2016

\begin{abstract}
Asymmetries in plasma density irregularity generation between the leading and trailing edges of the largescale plasma density structures in the high-latitude ionosphere are investigated. A model is developed that evaluates the gradient-drift instability (GDI) growth rate differences across the gradient reversal that is applicable at all propagation directions and for the broad range of altitudes spanning the entire lower ionosphere. In particular, the model describes asymmetries that would be observed by an oblique scanning radar near density structures in the polar cap such as elongated polar patches. The dependencies on the relative orientations between the directions of the gradient reversal, plasma convection, and wave propagation are examined at different altitudinal regions. At all altitudes, the largest asymmetries are expected for observations along the gradient reversals, e.g., when an elongated structure is oriented along the radar boresight. The convection direction that results in the strongest asymmetries exhibits a strong dependence on the altitude, with the optimal convection being parallel to the gradient reversal in the $\mathrm{E}$ region, perpendicular to it in the $\mathrm{F}$ region, and at some angle between these extremes in the transitional region. Implications for observations of polar patches by oblique scanning radars within the Super Dual Auroral Radar Network are discussed. It is demonstrated that the wave propagation direction relative to the prevalent convection and gradient directions plays a critical role in controlling both the irregularity growth rate and its asymmetries near gradient reversals.
\end{abstract}

Keywords. Ionosphere (modeling and forecasting; plasma waves and instabilities; polar ionosphere)

\section{Introduction}

Plasma density structures in the high-latitude ionosphere range in size from a few centimeters to hundreds of kilometers. In the ionospheric F region, they typically move with the large-scale plasma convection; i.e., their phase velocity is the $\boldsymbol{E} \times \boldsymbol{B}$ drift velocity. Observations of large-scale density structures in the polar cap ionosphere such as polar patches and Sun-aligned arcs have shown some asymmetry in the small-scale irregularity characteristics between their leading and trailing edges (e.g., Weber et al., 1984). In general, trailing edges exhibit stronger structuring, which is often interpreted in terms of more favorable conditions for the gradientdrift instability (GDI) along the trailing edge (Weber et al., 1984; Milan et al., 2002; Koustov et al., 2012; Moen et al., 2012).

The GDI growth rate is generally dependent on the relative directions of the background density gradient vector $\nabla n_{0}$ and the convection electric field $\boldsymbol{E}$ or, equivalently, the convection velocity $\boldsymbol{V}_{E}=\boldsymbol{E} \times \boldsymbol{B} / B^{2}$. It is widely accepted that the maximum GDI growth rate in the $F$ region occurs when the density gradient $\nabla n_{0}$ is parallel to the convection drift velocity $\boldsymbol{V}_{E}$ (Keskinen and Ossakow, 1982), while in the E region the maximum occurs when $\nabla n_{0} \| \boldsymbol{E}$. An analytic expression for the GDI growth rate in the $\mathrm{F}$ region has been derived in numerous studies, with most of these derivations considering a particular subset of mutual orientations between $\nabla n_{0}, \boldsymbol{V}_{E}$, and the wave vector $\boldsymbol{k}$. This typically results in the upper limit of the GDI growth rate of $\gamma=V_{E} / L$, where $L$ is a characteristic gradient scale length defined through $L^{-1} \equiv\left|\nabla n_{0}\right| / n_{0}$. More general vector configurations and growth rate expressions in the $\mathrm{F}$ region have also been considered (Keskinen and Ossakow, 1982; Makarevich, 2014), but their implications for studying the asymmetries between leading and trailing edges of large-scale structures have not been investi- 
gated. Further, it has been demonstrated recently that the GDI growth rate patterns change dramatically between the $\mathrm{E}$ and $\mathrm{F}$ regions (Makarevich, 2014). This means that one expects different signatures of small-scale irregularities and their asymmetries near large-scale structures at different altitudinal regions.

Oblique-sounding coherent radars within the Super Dual Auroral Radar Network (SuperDARN) routinely detect small-scale irregularities in a broad range of altitudes and directions (Chisham et al., 2007). Importantly, both E- and F-region irregularities are observed by the same radar (e.g., recent papers by Lamarche and Makarevich, 2015; Forsythe and Makarevich, 2015) and measurements of the background convection velocity $\boldsymbol{V}_{E}$ are routinely derived from F-region observations. In addition to their ability to observe largescale structures in the context of background plasma convection, SuperDARN radars provided important observational evidence in support of the GDI mechanism being responsible for the irregularity formation and asymmetries near gradient reversals (Milan et al., 2002; Koustov et al., 2012). Because these radars sample a particular range of the wave vector directions $\boldsymbol{k}$, the issue of the growth rate dependence on $\boldsymbol{k}$ is of critical importance. In particular, it is largely unknown if there are specific $\boldsymbol{k}$ directions or, equivalently, specific radar orientations that are more favorable for observing large-scale ionospheric structures and asymmetries between their leading and trailing edges.

The aim of this study is to investigate the asymmetries between the GDI growth rates near gradient reversals that often occur in the vicinity of large-scale density structures. The specific objectives are to (1) develop a quantitative approach for comparing the growth rates on either side of the structure, (2) analyze how the growth rates and asymmetries change for different orientations of the background density gradient and convection velocity and determine if there is an optimal radar orientation for which the asymmetry is the strongest, and (3) investigate how the growth rate and asymmetries change between the ionospheric $\mathrm{E}$ and $\mathrm{F}$ layers.

\section{Modeling of growth rates within radar's field of view}

In this study, we investigate behavior of the GDI growth rate within the field of view (FoV) of an oblique scanning radar with a particular focus on asymmetries between the leading and trailing edges of large-scale density structures in the ionosphere. Since growth rate exhibits a strong dependence on the altitude through its dependence on the ratio of the Hall to Pedersen conductivities $R=\sigma_{\mathrm{H}} / \sigma_{\mathrm{P}}$ (Makarevich, 2014), it is important to understand and model altitudinal dependencies of related parameters.

Figure 1a presents altitudinal dependencies of several parameters of interest. Shown are the ratios of collision frequencies and gyrofrequencies for electrons $-r_{\mathrm{e}}=v_{\mathrm{e}} /\left|\Omega_{\mathrm{e}}\right|$ (blue) and ions $r_{\mathrm{i}}=v_{\mathrm{i}} / \Omega_{\mathrm{i}}$ (green), the anisotropy parameter $\psi=-r_{\mathrm{i}} r_{\mathrm{e}}$ (pink), and the conductivity ratio $R=\sigma_{\mathrm{H}} / \sigma_{\mathrm{P}}=$ $\left(r_{\mathrm{i}}+r_{\mathrm{e}}\right) /(1+\psi)$ (dashed black line on top of the green one). The above notations are the same as in the recent study by Makarevich (2014), where the electrons gyrofrequency $\Omega_{\mathrm{e}}=-|e| B / m_{\mathrm{e}}$ and the ratio $r_{\mathrm{e}}=v_{\mathrm{e}} / \Omega_{\mathrm{e}}$ are negative, while other quantities of interest are positive. The $x$ axis in Fig. 1a does not have any units since all presented parameters are dimensionless quantities.

The collision frequencies used to calculate these parameters were derived from atmospheric neutral densities found from the Mass Spectrometer - Incoherent Scatter Extended (MSISE) model. Standard expressions for collision frequencies given by Schunk and Nagy (1978) were used. MSISE-90 is an empirical model of the densities and temperatures of neutral species in the Earth's atmosphere (Hedin, 1991). For this study, the neutral densities were found for 15 February 2014 at 20:00 UT at McMurdo Station, Antarctica $\left(77.88^{\circ} \mathrm{S}, 166.73^{\circ} \mathrm{E}\right.$, geographic); however the parameters considered are expected to be relatively stable, so this selection does not constrain the results.

We develop a model that is based on a typical observational geometry of individual SuperDARN radars. Each radar within SuperDARN scans through 16 look directions (radar beams) separated by $3.25^{\circ}$ in azimuth and receives returns from 75-100 range gates separated by $45 \mathrm{~km}$ (Chisham et al., 2007). For an oblique-sounding radar like SuperDARN, different range gates refer to different altitudes, with a virtual height model normally used to characterize this altitudinal dependence of the radar scattering volume as a function of the slant range. Figure $1 \mathrm{~b}$ shows the standard $\mathrm{Su}-$ perDARN virtual height model (Chisham et al., 2008). The ranges where the beam is within the $\mathrm{E}$ region (red), $\mathrm{F}$ region (blue), and transitional region (green) are indicated. The virtual height model assumes a particular height depending on the slant range. The model is based on where the signal is typically coming from for any given range. In the standard model the virtual height is constant throughout the $\mathrm{E}$ and $\mathrm{F}$ regions, but it changes rapidly in the transitional region between 600 and $800 \mathrm{~km}$ as shown in Fig. 1b. For individual observations in each beam, the height variations will be more gradual, but on average it is represented well by the model shown in Fig. 1b. A revised virtual height model in which virtual height changes with slant range has also been developed (Chisham et al., 2008), but since in the current study we were interested in the differences between the Eand F-region behavior (rather than small differences within each region), a standard virtual height model has been used.

Figure 1c presents a typical observational geometry in the horizontal plane. Here we show a FoV of the radar at McMurdo Station in Antarctica with the geographic South Pole located at the bottom left of panel c (in the center of concentric circles that show geographic parallels). The same model can be used for any SuperDARN radar in exactly the same way, with the only reason why McMurdo radar is presented here being that this radar often observes large-scale structures that are of interest to this study (Bristow et al., 2011; 

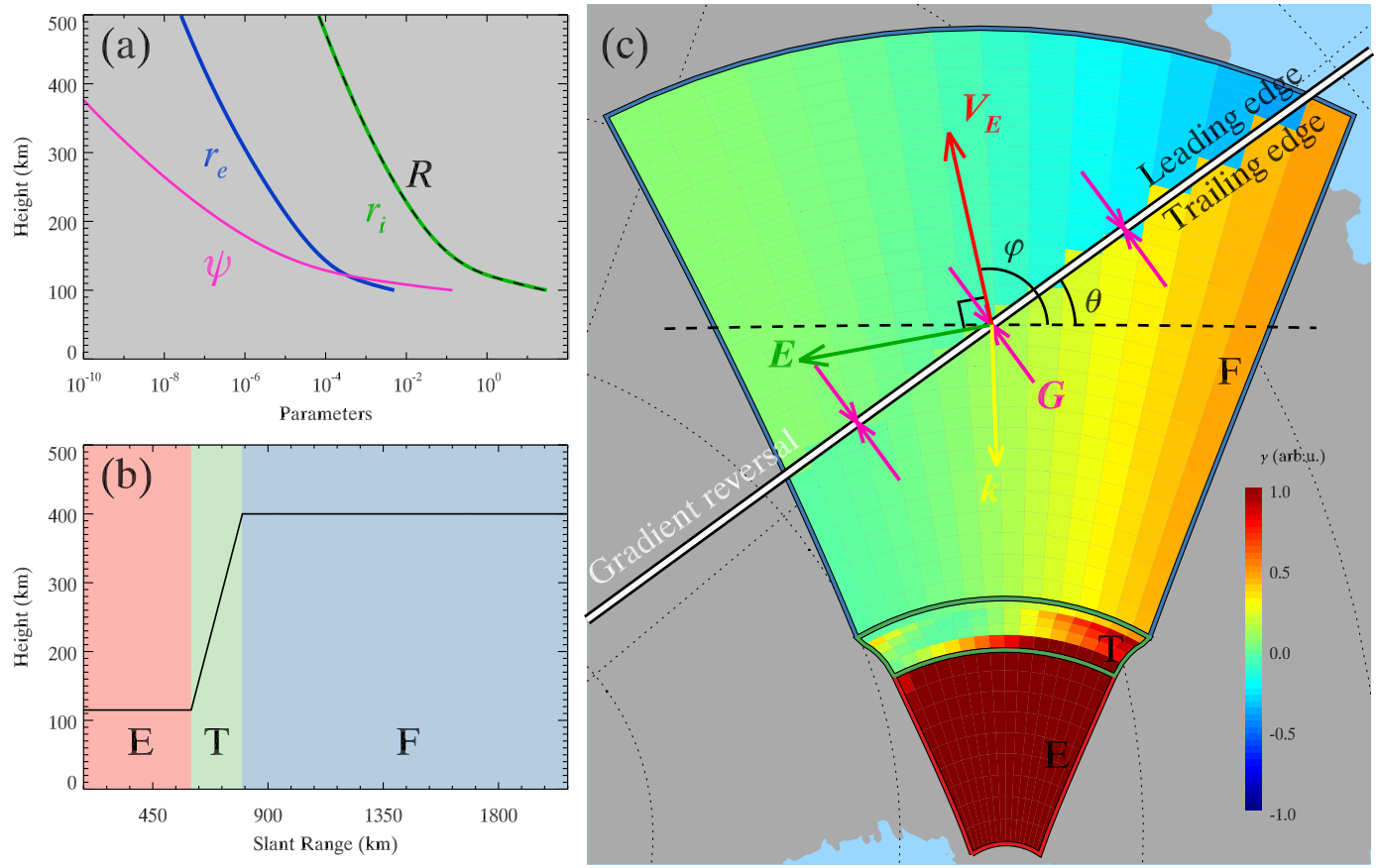

Figure 1. (a) Altitudinal variations of parameters of interest. Shown are the ratios of collision frequencies and gyrofrequencies for electrons $-r_{\mathrm{e}}=v_{\mathrm{e}} /\left|\Omega_{\mathrm{e}}\right|$ (blue) and ions $r_{\mathrm{i}}=v_{\mathrm{i}} / \Omega_{\mathrm{i}}$ (green), the anisotropy parameter $\psi=-r_{\mathrm{i}} r_{\mathrm{e}}$ (pink), and the conductivity ratio $R=\sigma_{\mathrm{H}} / \sigma_{\mathrm{P}}=$ $\left(r_{\mathrm{i}}+r_{\mathrm{e}}\right) /(1+\psi)$ (dashed black line on top of the green one). (b) Virtual height of radar scattering volume vs. the slant range. The areas shaded with red, green, and blue color refer to the E region, transitional region, and F region, respectively. (c) Geometry of observations and the gradient-drift instability (GDI) growth rate pattern in the radar's field of view (FoV). The plasma density gradient reversal is shown by the heavy white line. The gradient vectors $\boldsymbol{G}=\nabla n_{0} / n_{0}$ point towards the reversal in all points within the FoV as shown by representative pink vectors. Also shown are the angle $\theta$ between the gradient reversal and the horizontal axis (dashed black line), and the angle $\phi$ between the convection velocity direction $\boldsymbol{V}_{E}$ (red arrow) and the horizontal axis. The electric field $\boldsymbol{E}$ and the wave vector $\boldsymbol{k}$ are shown by the green and yellow arrows, respectively. The normalized GDI growth rate at every cell in the radar's FoV was calculated, and the radar cells are colored accordingly, with the color bar shown in the bottom-right corner. The E region, transitional region, and $\mathrm{F}$ region are outlined in red, green, and blue, respectively.

Lamarche and Makarevich, 2015). Other high-latitude radars in the network also observe these structures including the Hankasalmi (Milan et al., 2002) and Rankin Inlet (Koustov et al., 2012) radars in the Northern Hemisphere, and the present results can also be used to interpret these observations.

Figure 1c presents a fan plot of the McMurdo radar's FoV with a large-scale structure across the FoV (thick white line). It it assumed that the field of gradient vectors $\boldsymbol{G}=\nabla n_{0} / n_{0}$ is uniform in magnitude, with directions always pointing towards the structure throughout the FoV. In other words, the direction of the density gradient reverses at this structure, which will be referred to as the gradient reversal for the remainder of this paper. An example of a structure near which such a reversal may occur is a Sun-aligned arc. Three representative pairs of the gradient vector $\boldsymbol{G}$ are shown with pink arrows.

In order to quantify effects due to different structure orientations and motion directions, two angles were used. The first angle describes the structure orientation relative to the radar. This is the angle $\theta$ between the gradient reversal direction and the direction perpendicular to the boresight (FoV center direction); this perpendicular direction is shown by the dashed horizontal line in Fig. 1c. The second angle $\phi$ is the angle between the structure drift velocity (red vector in Fig. 1c) and the dashed horizontal line. Since these structures tend to move with the background convection velocity (Makarevich et al., 2015), this drift velocity is simply $\boldsymbol{V}_{E}=\boldsymbol{E} \times \boldsymbol{B} / B^{2}$. The leading and trailing edges of the structure are determined by the direction of $\boldsymbol{V}_{E}$. The direction of the electric field $\boldsymbol{E}$ (green vector) is also defined by $\phi$ because $\boldsymbol{E}$ is perpendicular to $\boldsymbol{V}_{E}$. The magnetic field, $\boldsymbol{B}$, is assumed to be straight out of the page, which is approximately consistent with the orientation of the Earth's magnetic field in the southern polar cap, and so $\boldsymbol{E}$ is simply rotated counterclockwise by $90^{\circ}$ from $\boldsymbol{V}_{E}$. The direction of the wave vector $\boldsymbol{k}$ (yellow vector) is determined by the location of the point within the radar FoV, as $\boldsymbol{k}$ will be opposite to the direction of the radar beam. 
Finally, the GDI growth rates were found for each radar beam-range cell as follows. The location and height of the center of each radar cell was found with a standard positioning method utilizing virtual heights shown in Fig. 1b. The height was used to find the value of the conductivity ratio $R$ using information presented in Fig. 1a. Using these quantities, the GDI growth rate at any point within the radar FoV can be calculated (Makarevich, 2014, Eq. 18) using

$$
\begin{aligned}
\gamma & =\frac{G V_{E}}{1+\psi}(\hat{\boldsymbol{k}} \cdot \hat{\boldsymbol{b}} \times \hat{\boldsymbol{g}})(\hat{\boldsymbol{k}} \cdot \hat{\boldsymbol{e}}-R \hat{\boldsymbol{k}} \cdot \hat{\boldsymbol{e}} \times \hat{\boldsymbol{b}}) \\
& =\frac{G V_{E}}{1+\psi} \gamma^{\prime} .
\end{aligned}
$$

Here, $\hat{\boldsymbol{k}}, \hat{\boldsymbol{b}}, \hat{\boldsymbol{g}}$, and $\hat{\boldsymbol{e}}$ represent unit vectors in the direction of the wave vector $\boldsymbol{k}$, magnetic field $\boldsymbol{B}$, density gradient $\boldsymbol{G}$, and electric field $\boldsymbol{E}$, respectively. The quantity $G V_{E}$ will be the same for all vector orientations and heights. The anisotropy parameter $\psi$ will change at different heights, but it is always small relative to 1 (Fig. 1a) so its effect is minimal. Since this study focuses primarily on different relative orientations of the gradient and convection directions and altitude regimes, it is convenient to use a normalized growth rate $\gamma^{\prime}$. For the remainder of this paper, the GDI growth rate will refer to the normalized growth rate $\gamma^{\prime}$ in arbitrary units, with the prime symbol omitted for brevity.

Each radar cell in Fig. 1c is color-coded in the GDI growth rate at its center, with the color bar given in the bottom-right corner of Fig. 1c. On the left side of the radar FoV, the growth rate is positive on the leading edge of the gradient reversal and negative on the trailing edge, but both are very close to 0 . Conversely, the growth rate is negative on the leading edge and positive on the trailing edge on the right side of the FoV. In addition, both the negative and positive values are much larger $(\sim \mp 0.5)$ than on the left side. For this presentation, a gradient field that is uniform in magnitude was assumed; this was done to illustrate the most salient features of the GDI growth rate pattern and in particular effects due to variations in direction and altitude. In practice, the gradient magnitude will also change depending on the distance from the structure, but the directional and altitudinal effects in asymmetries will be the same. This is further examined in Sect. 3, where a more realistic approach is employed. The important common features are that the GDI growth rate and its asymmetries are strongly dependent on the altitude and direction of observations. The direction of observation is directly related to the wave propagation direction since for every beam the radar samples a narrow range of propagation directions. The present study has considered a dependence on the observational direction, but an alternative presentation of the same effect is to consider a fixed scattering volume with variable propagation directions. This alternate presentation has been shown in Makarevich (2014), their Figs. 1 and 2. These figures show the GDI growth rate in all wave vector directions for set gradient and drift velocity directions for the $\mathrm{E}$ and F regions, respectively.

Figure 1c presented the growth rate pattern for one particular orientation of the gradient reversal and plasma convection. Figure 2 presents the same analysis for four different orientations and locations of the gradient reversal. In all cases, the convection direction is assumed to be perpendicular to the gradient reversal direction $\phi-\theta=90^{\circ}$. This is because large-scale structures, such as polar patches, tend to drift perpendicular to their elongation direction (Makarevich et al., 2004, 2015). This requirement was convenient for presenting different growth rate patterns but will be relaxed later in this study.

Figure 2a shows a gradient reversal through the center of the F-region portion of the FoV that is perpendicular to the boresight $\theta=0^{\circ}$. The growth rate on both sides of the gradient reversal is small (close to 0 ), but it is slightly negative on the leading edge and slightly positive on the trailing edge. This difference is more evident near the edge beams of the FoV. In the transitional region, the growth rate is again close to 0 near the boresight but increases to $\sim 0.7$ on either edge of the beam. The E region shows a completely different pattern, where the growth rate is negative on the left side and smoothly transitions to positive on the right side. In addition, the most extreme values for growth rate in the E region are above \pm 1.0 , i.e., much higher than what is observed in the $\mathrm{F}$ region.

Figure $2 b$ shows an inclined gradient reversal through the FoV in the $\mathrm{F}$ region $\theta=40^{\circ}$. Again, the growth rate is negative on the leading edge and positive on the trailing edge, but the difference is much more dramatic. The growth rate depends on $\boldsymbol{k}$, so the magnitude of difference across the reversal again changes for different beams. This results in a much greater contrast in the growth rate on the right side of the FoV. The normalized growth rate is -0.7 on the leading edge and +0.7 on the trailing edge. This is in contrast with the difference on the FoV left side, where the values are only $\sim \mp 0.1$. The transitional region shows a similar pattern with lower positive values on the left side and growth rate above 1.0 on the right side. The growth rate is above 1.0 throughout the $\mathrm{E}$ region as well.

Figure $2 c$ presents the same configuration as Fig. 2a except that a gradient reversal is centered in the transitional region. Similar to Fig. 2a, the growth rate is close to 0 on both sizes of the reversal in the center of the radar FoV. However, there are more extreme values $(\sim \mp 0.5)$ near the edge beams. Figure $2 \mathrm{~d}$ shows an inclined gradient reversal in the transitional region. The growth rate difference across the gradient is again more extreme further away from the boresight. Overall, this analysis demonstrated that both the gradient reversal orientation and its position in the FoV are important in controlling the asymmetries between the leading and trailing edges. 


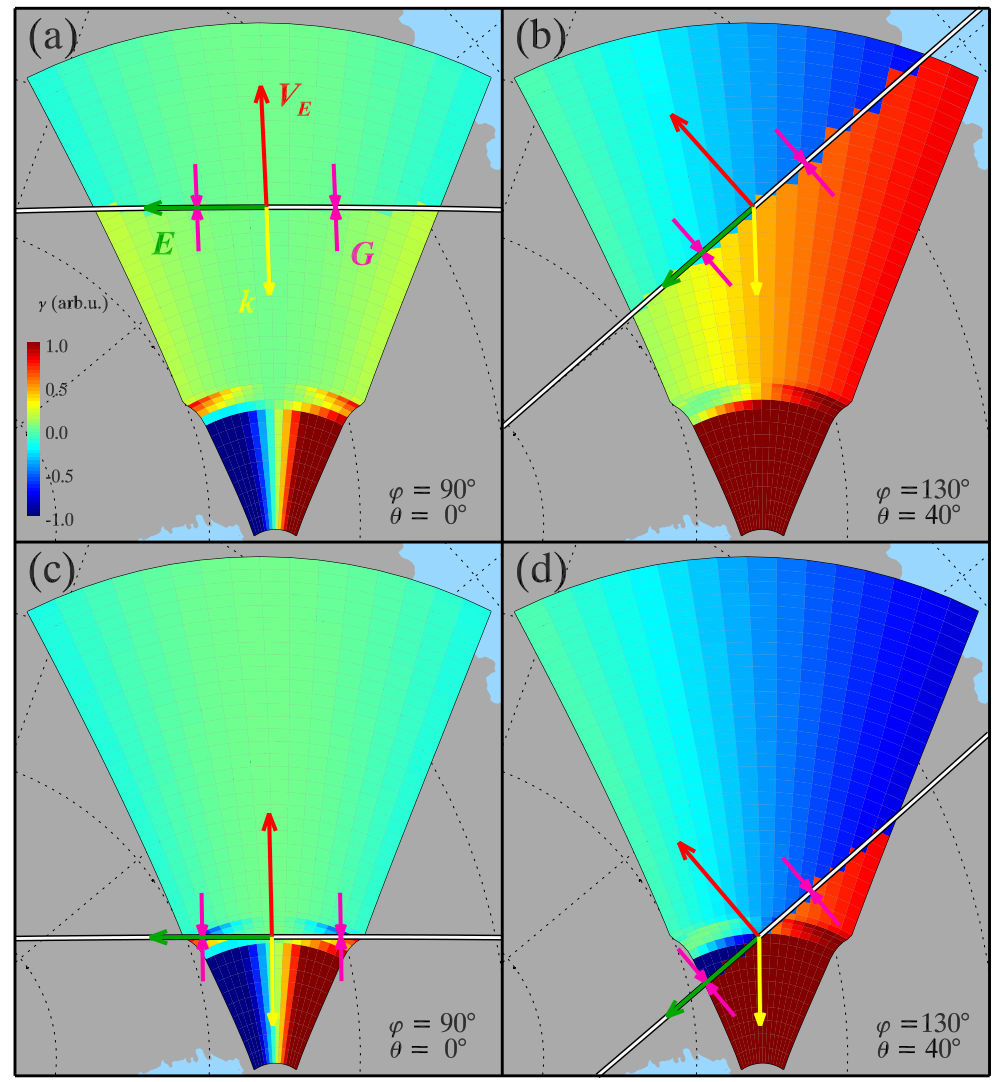

Figure 2. The GDI growth rate within the radar's FoV for four cases of gradient reversal orientation and position (white line). In all cases, the convection direction $\boldsymbol{V}_{E}$ (red arrow) is perpendicular to the gradient reversal direction. The orientation of the vectors $\boldsymbol{G}, \boldsymbol{V}, \boldsymbol{E}$, and $\boldsymbol{k}$ is shown in each panel, using the same colors as in Fig. 1c. The GDI growth rate at each radar cell is given by the cell color, with the color bar given in the bottom-left corner of (a). (a) and (b) show a gradient reversal centered in the F region, while (c) and (d) show the reversal in the transitional region. The angles $\theta$ and $\phi$ (as defined in Fig. 1c) are also given in the bottom-right corner of each panel.

\section{Modeling of growth rate differences across the gradient reversal}

In order to quantify the above-noted differences in the growth rate for different orientations of the gradient reversal, the average change in the growth rate across the reversal $\Delta \gamma_{\text {avg }}$ was found for any general configuration of the gradient reversal and the convection direction. The approach presented in Figs. 1c and 2 was useful for presenting and emphasizing general spatial patterns in the growth rate. In this section, we employ two new methods that are more robust, independent of the way the radar FoV is divided into beam-range cells, and more suitable for evaluating the growth rate differences in the vicinity of large-scale structures. These two new methods are described below.

\subsection{Numerical method}

In the first method, evenly spaced points were selected along the gradient reversal within the radar FoV. These points did not necessarily correspond to real range gates or beams used in the standard SuperDARN radar configuration. A virtual radar beam was constructed that intersected each point. From this, the virtual slant range and beam azimuth were found, which were used to find the wave vector $\boldsymbol{k}$ and the height corresponding to that point. The growth rates on opposite sides of the gradient reversal were found as follows. For the leading edge, the gradient vector $\boldsymbol{G}$ is set to be perpendicular to the gradient reversal and in a direction such that the component of $\boldsymbol{V}_{E}$ along $\boldsymbol{G}$ is negative, i.e., $\boldsymbol{V}_{E} \cdot \boldsymbol{G}<0$. For the trailing edge, the opposite direction is selected such that $\boldsymbol{V}_{E} \cdot \boldsymbol{G}>0$. These two cases can be thought of as the limiting cases when two points on either side of the reversal are both moved towards the reversal until they are both colocated on it. The GDI growth rate for both cases can then be calculated using Eq. (1) as described above. The difference in growth rate across the reversal, $\Delta \gamma$, can then be found by subtracting the trailing-edge case from the leading-edge case. The average difference in growth rate across the reversal for the entire FoV, $\Delta \gamma_{\text {avg }}$, can be found by averaging $\Delta \gamma$ of every point initially considered along the gradient reversal. 


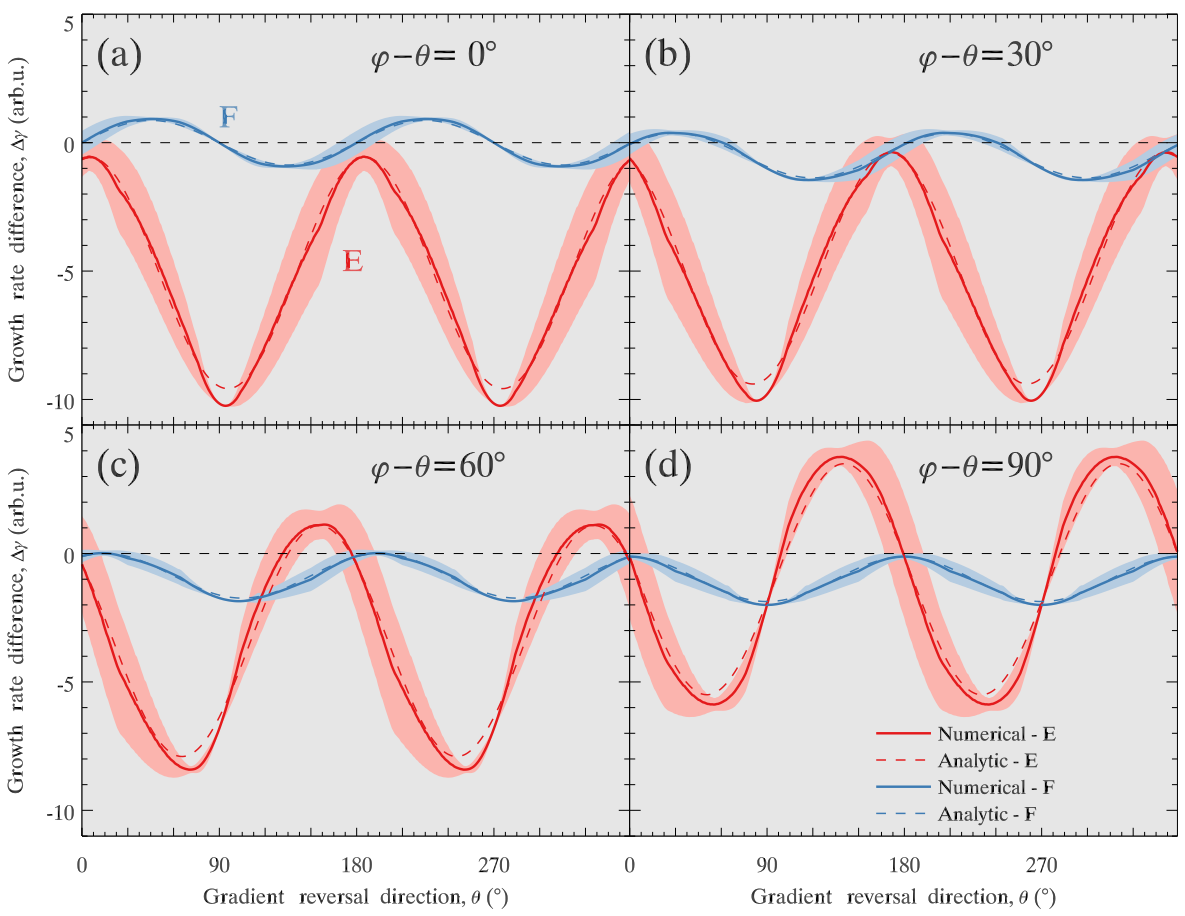

Figure 3. The difference in the GDI growth rate across the gradient reversal for four selected angles between the reversal and convection direction. The gradient reversal direction $\theta$ is on the $x$ axis, and the average growth rate difference across the reversal $\Delta \gamma$ is on the $y$ axis for each panel. The difference between $\phi$ and $\theta$ is given in the top-right corner of each plot. The difference in the average growth rate $\Delta \gamma$ computed using a numerical approach (see text for details) is shown by the solid lines, with the shaded regions representing the standard deviation. The dashed lines show the behavior of $\Delta \gamma$ calculated using an analytic expression. The trends for both the E-region (red) and F-region (blue) cases are shown in each panel.

\subsection{Analytic method}

An alternative approach is to develop an analytic expression for $\Delta \gamma_{\text {avg }}$ for any general direction of the gradient reversal $\theta$ and convection $\phi$ as described in Appendix A. The result is

$$
\begin{aligned}
\Delta \gamma_{\mathrm{avg}}= & H(\theta, \phi)[\sin (\phi-\theta)-C \sin (\phi+\theta) \\
& +R(\cos (\phi-\theta)-C \cos (\phi+\theta))],
\end{aligned}
$$

with

$$
\begin{aligned}
H(\theta, \phi)= & \Theta(\phi-\theta+\pi)-\Theta(\theta-\phi-\pi) \\
& +2 \Theta(\phi-\theta-\pi)-2 \Theta(\phi-\theta),
\end{aligned}
$$

function $\Theta$ being the Heaviside step function, and $C=0.87$ for SuperDARN observations (Appendix A). This expression can be readily used to compute $\Delta \gamma_{\text {avg }}$ for any given combination of $\theta$ and $\phi$. One limitation of this expression is, however, that it can only be applied when the conductivity ratio $R$ is approximately constant, i.e., in the $\mathrm{E}$ region or in the $\mathrm{F}$ region but not in the transitional region.

\subsection{General vector orientations}

The two methods for finding the average difference in the GDI growth rate across the gradient reversal can now be employed to characterize different orientations. In this section, we consider a more general case of different relative orientations of the gradient reversal and the convection direction, not necessarily restricted to the case of $\theta-\phi=90^{\circ}$ that was considered in Sect. 2. In other words, we consider all possible differences $\theta-\phi$.

Figure 3 examines the growth rate difference across the gradient reversal for four selected angle differences $\theta-\phi$ as indicated in the top-right corner of each panel. In each case, the average growth rate difference, notated simply by $\Delta \gamma$ for the remainder of this paper, is calculated for different $\theta$ values from 0 to $360^{\circ}$. In each plot, the convection direction $\phi$ changes with $\theta$ so that their difference remains constant. The average growth rate difference is shown on the $y$ axis in arbitrary units. The numerical method results (Sect. 3.1) are shown for the averages (solid lines) and standard deviations (shaded regions). The blue and red curves correspond to the E- and F-region cases. The analytic method results (Sect. 3.2) are also presented for the expected average differences (dashed lines). Because the growth rate difference is calculated by subtracting the trailing edge $\gamma$ from the leading edge $\gamma$, negative $\Delta \gamma$ indicates larger instability growth on the trailing edge than the leading edge. In addition, large $\Delta \gamma$ (either positive or negative) indicates a strong contrast between the two sides of the gradient reversal. 
Figure 3a shows a case of $\phi-\theta=0^{\circ}$, i.e., the case when the convection direction is parallel to the gradient reversal. This corresponds to $\boldsymbol{V}_{E} \perp \boldsymbol{G}$ or $\boldsymbol{E} \| \boldsymbol{G}$ on the trailing edge, which is the condition for most favorable gradient orientation in the E region (Makarevich, 2014). The growth rate difference in the $\mathrm{E}$ region (red) is negative at all gradient reversal directions $\theta$, and it changes with $\theta$ in a sinusoidal fashion. It has the lowest $\Delta \gamma$ minimum values observed out of all four cases $\sim-10$ at 90 and $270^{\circ}$. The average growth rate difference for the $\mathrm{F}$ region (blue) also shows a periodic behavior with $\theta$; it is positive for $0-90^{\circ}$ and $180-270^{\circ}$ and negative for all other angles. The amplitude of oscillations is much smaller than in the $\mathrm{E}$ region. The segments where $\Delta \gamma>0$ correspond to the growth rate on the leading edge exceeding that on the trailing edge. However, $\Delta \gamma \approx 1$ and $\Delta \gamma \approx-1$ are the maximum and minimum values, respectively, so the contrast across the gradient reversal is relatively small regardless of whether the leading or trailing edge has the greater growth rate. The important conclusion from this analysis is that the contrast or the asymmetry magnitude depends strongly on the structure orientation angle $\theta$, which confirms the previous results based on two angles $\theta$ only (Fig. 2a and b).

Figure $3 \mathrm{~b}$ and $\mathrm{c}$ present the same analysis but with a set angle of 30 and $60^{\circ}$, respectively, between the gradient reversal and the convection direction. These show the gradual transition between the extreme cases of the convection direction parallel to the gradient reversal (Fig. 3a) and the convection direction perpendicular to the reversal (Fig. 3d). The general E-region pattern starts to move towards more positive values, and the F-region pattern moves towards more negative values. The location of the peaks in growth rate difference changes as well. For the $\mathrm{E}$ region, the location of the lowest $\Delta \gamma$ moves gradually from $\theta \approx 90^{\circ}, 270^{\circ}$ when $\phi-\theta=0^{\circ}$ to $\theta \approx 45^{\circ}, 225^{\circ}$ when $\phi-\theta=90^{\circ}$. Similarly, for the $\mathrm{F}$ region, the location of the lowest $\Delta \gamma$ moves from $\theta \approx 135^{\circ}, 315^{\circ}$ to $\theta \approx 90^{\circ}, 270^{\circ}$ between these two situations.

Figure $3 \mathrm{~d}$ shows the case when the convection direction is perpendicular to the gradient reversal, which corresponds to $\boldsymbol{V}_{E} \| \boldsymbol{G}$. From previous studies, this is the condition where the growth rate on the trailing edge is the greatest in the $\mathrm{F}$ region. Similar to Fig. 3a, the growth rate difference in Fig. 3d for the $\mathrm{F}$ region (blue) is negative for all directions of the gradient reversal and has the lowest values observed for the F region $(\sim-2)$ at 90 and $270^{\circ}$. This corresponds to the largest contrast between the leading and trailing edge of the structure. Thus the largest difference is observed when the growth rate peaks. This is only true, however, for the largest difference, i.e., at a particular $\theta$ value where this largest magnitude is found. For other $\theta$ values, the difference may be larger or smaller than at other values of $\phi-\theta$. The E-region (red) extreme values (maximum $\Delta \gamma \approx 4$ and minimum $\Delta \gamma \approx-6$ ) have the lowest magnitude out of all four cases shown in panels a- $\mathrm{d}$. This indicates that the contrast in the E region is the smallest for this particular orientation, although it is still much larger than that observed in the $\mathrm{F}$ region.
Throughout all four cases, there are several common features. The amplitude of variations of the growth rate difference in the $\mathrm{F}$ region is consistently much less than that in the E region. Generally, there is very good agreement between the numerical method and the analytic method in both the $\mathrm{E}$ and $\mathrm{F}$ regions. Typically, the results from the analytic method fall well within 1 standard deviation of the numerical method. The exceptions to this occur when $\theta$ is 90 or $270^{\circ}$. The discrepancy in the results in these locations is due to the fact that the two methods have some inherent differences. In these cases, the gradient reversal structure passes through the radar FoV along its boresight or central beam, so only points along that line (and that azimuth) are considered for the numerical approach, while the whole range of azimuths in the analytic approach is still integrated over. For most gradient reversal orientations, however, this difference is negligible.

The analysis presented in Fig. 3 can be extended to include any arbitrary orientation between the gradient reversal and convection direction by allowing $\phi$ and $\theta$ to vary independently. Both the numerical method and the analytical method can be used to find the average difference in growth rate for any direction of the gradient reversal and plasma convection in the $\mathrm{E}$ and $\mathrm{F}$ regions. Figure 4 presents the results of this analysis in the form of contour plots of the growth rate difference $\Delta \gamma$ vs. angles $\theta$ and $\phi$. Figure $4 \mathrm{a}$ and $\mathrm{b}$ show the results of the analytic method in the $\mathrm{E}$ and $\mathrm{F}$ regions, respectively. The remaining four panels show the results from the numerical method in (c) the E region, (d) the F region, (e) the transitional region, and (f) all regions combined. With the exception of Fig. 4e, different values of $\Delta \gamma$ along the gradient reversal were included in the averaging only for the points with specified virtual heights, e.g., $115 \mathrm{~km}$ for Fig. 4c. In Fig. 4f, all points along the gradient reversal within the FoV were considered.

The discontinuities in $\Delta \gamma$ that occur on diagonal lines $\phi=\theta, \phi=\theta+180^{\circ}$, and $\phi=\theta-180^{\circ}$ are due to the fact that the convection velocity vector crosses the gradient reversal at these points and the leading and trailing edges of the gradient reversal switch. This feature is common in all panels in Fig. 4. On these discontinuities, the $\Delta \gamma$ values always switch their sign, but their magnitude is the same on both sides of the discontinuity. An alternative presentation would be to only show magnitude, but the current version also presents information about the sign and the sense of the change, which is also useful.

Figure 4a shows the average growth rate difference across the gradient reversal found by the analytic method in the $\mathrm{E}$ region. The same information but for four specific cases of $\phi-\theta$ was shown in Fig. 3. Specifically, each dashed red line in Fig. 3 represents one diagonal cross section in Fig. 4a. For example, the red dashed line in Fig. 3a refers to the main diagonal line $\phi=\theta$, and the red dashed line in Fig. 3d refers to the line $\phi=\theta+90^{\circ}$. The peak magnitudes in $\Delta \gamma$ occur at $\theta=90^{\circ}, 270^{\circ}$ and $\phi=90^{\circ}, 270^{\circ}$. This agrees with what is shown in Fig. 3a. Physically, this means that the largest con- 


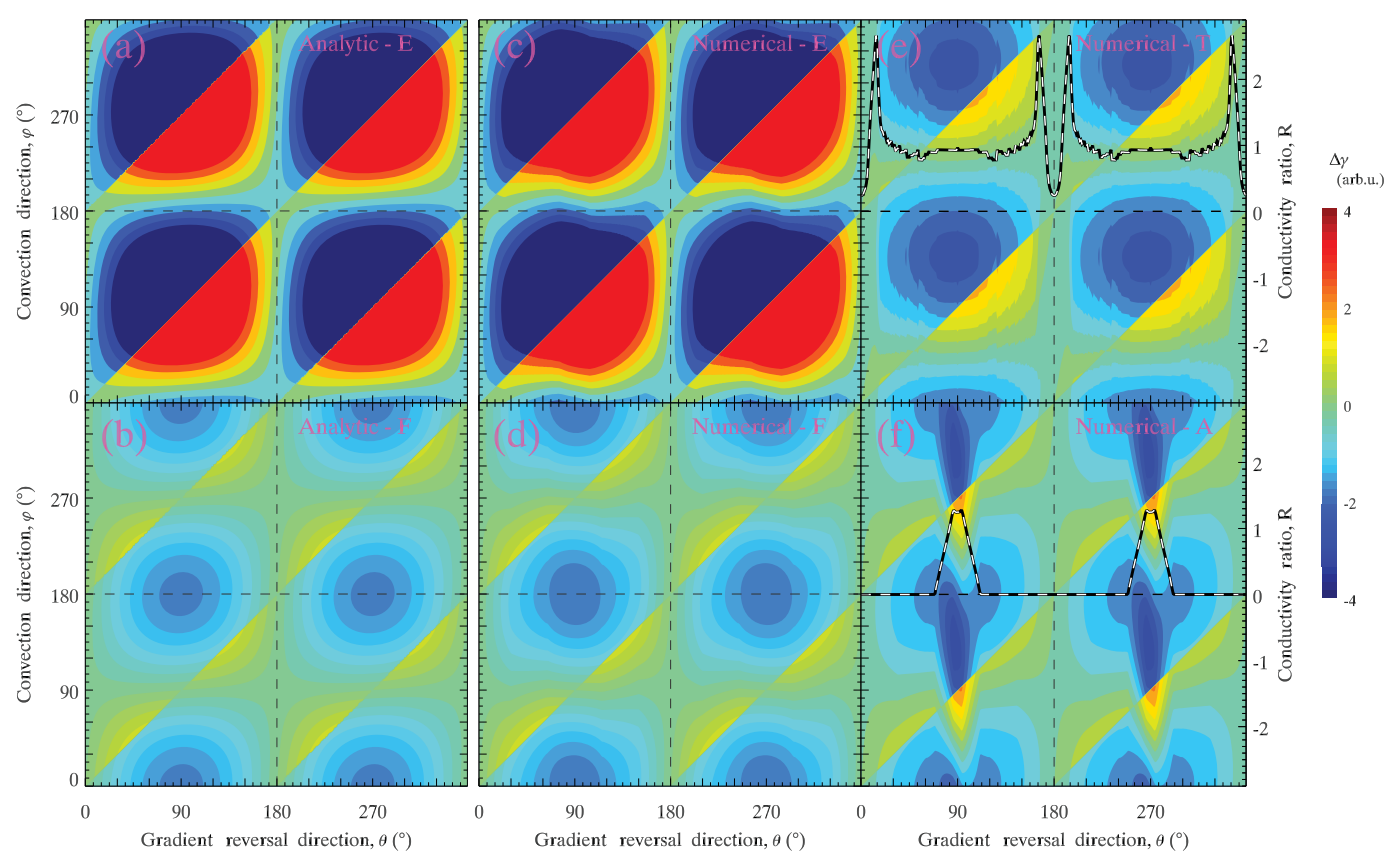

Figure 4. Contour plots of the growth rate difference $\Delta \gamma$ vs. the two angles of interest. The direction of the gradient reversal $\theta$ is on the $x$ axis, and the convection direction $\phi$ is on the $y$ axis. The color bar is given to the right. (a) and (b) show the results using an analytic approach in the $\mathrm{E}$ and $\mathrm{F}$ regions, respectively. (c-f) show the results using a numerical approach in the E region, the $\mathrm{F}$ region, the transitional region, and all regions, respectively. The dashed black-and-white line in (c) and (f) indicates the average value of the conductivity ratio $R$ along the gradient reversal for different angles $\theta$ in the transitional region and all regions, respectively. The scale for $R$ is shown on the right of (c) and (f).

trast in growth rate across the gradient reversal occurs when the reversal is passing through the radar FoV along the boresight and convection is parallel or antiparallel to the reversal. The lowest magnitudes of $\Delta \gamma$ occur approximately at the lines $\theta=0^{\circ}, \theta=180^{\circ}, \phi=0^{\circ}$, and $\phi=180^{\circ}$. At these locations the difference is close to 0 (green color). This means that contrast in the growth rate is the least if either the gradient reversal or the convection direction is perpendicular to the boresight.

Figure $4 \mathrm{~b}$ presents the same information but for the $\mathrm{F}$ region. This refers to the blue dashed lines in Fig. 3. In this case, the peak magnitudes in $\Delta \gamma$ occur at $\theta=90^{\circ}, 270^{\circ}$ and $\phi=0^{\circ}, 180^{\circ}$. This means that the greatest contrast in growth rate occurs when the gradient reversal is parallel to the boresight and the convection is perpendicular to it. The smallest magnitudes of $\Delta \gamma$ occur at the lines $\theta=0^{\circ}, \theta=180^{\circ}$, $\phi=90^{\circ}$, and $\phi=270^{\circ}$. This means that the least contrast across the reversal will occur when the gradient reversal is perpendicular to the boresight or when the convection direction is either parallel or antiparallel to the radar boresight.

Figure $4 \mathrm{c}$ and $\mathrm{d}$ present the growth rate difference in the $\mathrm{E}$ and $\mathrm{F}$ regions, respectively, found with the numerical method. A comparison of Fig. $4 a$ and $b$ with Fig. $4 c$ and d shows that the results of the analytic and numerical methods are very similar for both the $\mathrm{E}$ and $\mathrm{F}$ regions. The results for the numerical method (Fig. 4c and d) are not as smooth as the analytic method results, but this is simply due to the discrete steps that are used in the numerical method. Comparison between the $\mathrm{E}$ and $\mathrm{F}$ region patterns, however, reveals a few interesting features. First, the location of the minima moves from $\phi=90^{\circ}, 270^{\circ}$ in the E region to $\phi=0^{\circ}, 180^{\circ}$ in the $\mathrm{F}$ region. These results agree with what was observed in Fig. 3. In addition, there are large regions of strongly positive $\Delta \gamma$ (dark red) in the $\mathrm{E}$ region (Fig. $4 \mathrm{a}$ and c) that do not appear in the $\mathrm{F}$ region. There are some areas of positive $\Delta \gamma$ in the $\mathrm{F}$ region, but they are much more limited and have a maximum value of only $\sim 1$. This means that, while there are significant areas where the growth rate on the leading edge is greater than that on the trailing edge in the E region, the growth rate on the trailing edge almost always exceeds the leading edge in the $\mathrm{F}$ region. This is an important generalization of the well-known result that growth rates are more positive on the trailing edge, where $\boldsymbol{G} \cdot \boldsymbol{V}_{E}>0$.

Figure $4 \mathrm{e}$ shows results of the numerical analysis of the transitional region. This panel does not have a comparison with the analytic method because the analytic method requires a constant conductivity ratio $R$, which changes rapidly from $\sim 5.08$ in the E region to 0.001 in the F region (Fig. 1a). To demonstrate this, the average $R$ value along the gradient reversal is plotted using the black-and-white dashed line and the scale shown on the right. The ratio depends on the gradient reversal direction $\theta$, but not on the convection di- 
rection $\phi$. As angle $\theta$ changes, the gradient reversal passes through different portions of the FoV with different heights. Different altitudes correspond to hugely different values of $R$, which causes the variation in the conductivity ratio shown in Fig. 4e. On average, however, the conductivity ratio is somewhere in between its values in the $\mathrm{E}$ and $\mathrm{F}$ regions, so the pattern shown is between the E-region pattern and F-region pattern. The minimum is between $\phi=90^{\circ}$ (the Eregion value) and $\phi=180^{\circ}$ (the F-region value) and has a value of $\sim-3$. This will be further examined in Fig. 5 .

Finally, Fig. $4 \mathrm{f}$ presents the same analysis for all regions considered together, i.e., with averaging done over all points along the gradient reversal regardless of their virtual height. For most angles of the gradient reversal, only the $\mathrm{F}$ region will be sampled (Fig. 1c). The gradient reversal will only pass through the transitional and $\mathrm{E}$ region if it is close to parallel with the boresight, i.e., when $\theta$ is close to either 90 or $270^{\circ}$. This is why the majority of the pattern in Fig. $4 \mathrm{f}$ resembles the F-region patterns (Fig. 4d) but changes sharply around $\theta=90^{\circ}$ and $\theta=270^{\circ}$ due to contributions from the E-region pattern (Fig. 4c). This can also be seen in the conductivity ratio. For most angles, average $R$ only has contribution from the $\mathrm{F}$ region, so it is at the F-region value (very close to 0 on this scale). However, close to $\theta=90^{\circ}$ and $\theta=270^{\circ}$ average $R$ includes E-region values ( $\left.\sim 5\right)$, so it increases sharply. This causes $\Delta \gamma$ to be relatively low with values between -1.5 and +1.5 for most angles $\theta$; it changes around $\theta=90^{\circ}$ and $\theta=270^{\circ}$ to have values less than -2 . Overall, this analysis shows that both the peak asymmetry and where it is seen in $\theta$ and $\phi$ change significantly with altitude.

\subsection{Behavior in the transitional region}

Figure 5 shows a more detailed analysis of the transitional region. The transitional region was divided into segments that were $2 \mathrm{~km}$ in slant range. In each of these segments, the analysis presented in Fig. 4 was performed and the average height and average conductivity ratio were found. Figure 5a shows the average conductivity ratio (black line) and height (orange line) from 550 to $850 \mathrm{~km}$ in slant range. The ranges where the beam is in the $\mathrm{E}$ (red), $\mathrm{F}$ (blue), and transitional (green) regions are shaded. Both the height and the conductivity ratio are constant in the $\mathrm{E}$ and $\mathrm{F}$ regions since virtual height is constant at these ranges in the standard virtual height model (Fig. 1b). The height increases linearly in the transitional region, while the conductivity ratio decreases rapidly between 600 and $616 \mathrm{~km}$ (shaded in yellow) at the lowest ranges within the transitional region. The $R$ value continues to decrease throughout the transitional region, but above $\sim 620 \mathrm{~km}$ in the slant range it is so close to 0 that further changes do not affect the results appreciably. The region highlighted in yellow, where most of the change in $R$ occurs, is examined more closely in Fig. $5 \mathrm{~b}$ and c.
Figure $5 \mathrm{~b}$ has the same format as Fig. 5a, but it focuses on the $16 \mathrm{~km}$ wide region in the slant range highlighted with yellow. In these $16 \mathrm{~km}$, the conductivity ratio (black line) decreases by approximately 1 order of magnitude, from $\sim 5$ to 0.5 . The height (orange line) increases from $\sim 110$ to $130 \mathrm{~km}$. These changes make a large difference in the patterns of difference of growth rate, as described below.

Each small panel in Fig. 5c has the same format as Fig. 4a. The top row shows the numerical analysis, and the bottom shows the analytic analysis of each $2 \mathrm{~km}$ segment. The value of $R$ for the analytic method was taken from the average $R$ for each $2 \mathrm{~km}$ segment, as shown in Fig. 5b. From 600 to $602 \mathrm{~km}, R$ is close to the E-region value $(\sim 5.1)$, so the patterns for $\Delta \gamma$ are close to those for the E region (Fig. 4a and c). However, by $614-616 \mathrm{~km}$, the value of $R$ has decreased substantially, and the patterns resemble the F-region patterns (Fig. 4b and d) much more closely. From $600 \mathrm{~km}$ to $616 \mathrm{~km}$, the location of lowest value of $\Delta \gamma$ changes from $\phi=90^{\circ}, 270^{\circ}$ to $\phi=0^{\circ}, 180^{\circ}$. This represents the change from where the lowest $\Delta \gamma$ values are in the E region to their location in the $\mathrm{F}$ region. In addition, the lowest value of $\Delta \gamma$ also increases from less than -8 (E region) to $\sim-3$ (F region). The areas of highly positive $\Delta \gamma$ that appeared in the E region (Fig. 4a and c) are not evident above $606 \mathrm{~km}$. This indicates that, after this point, there is no longer a significant subset of orientations where the growth rate on the leading edge is larger than that on the trailing edge.

\section{Discussion}

In this study, we have applied the GDI linear fluid theory to study the asymmetries in the GDI growth rate on either side of a large-scale density structure, particularly as applied to observations with oblique scanning radars such as SuperDARN. The important new aspect of this study is explicit consideration of varying wave vector direction and altitude and their effects on asymmetries in the plasma structuring processes. We focus on the first-order effects due to the differences in the GDI growth rate that are often involved in interpreting asymmetries in intensity and occurrence characteristics of small-scale plasma irregularities in the highlatitude ionosphere. Below, we discuss the applicability of these results and their implication for interpretation of radar observations near gradient reversals.

The original expression used to define the GDI growth rate (Eq. 1) has been derived under the assumption of negligible inertial effects (Makarevich, 2014), i.e., in the low-frequency and long-wavelength limit: $\omega \ll v_{i n}, \lambda \gg 2 \pi V_{E} v_{i n}^{-1}$. The fluid theory itself also applies directly to long wavelengths, i.e scales much larger than the ion mean free path. The observations with which we compare our results refer to smallscale irregularities and, more specifically, decameter-scale waves in the $\mathrm{E}$ and $\mathrm{F}$ regions as well as in the transitional region, in the case of SuperDARN observations. In the E re- 

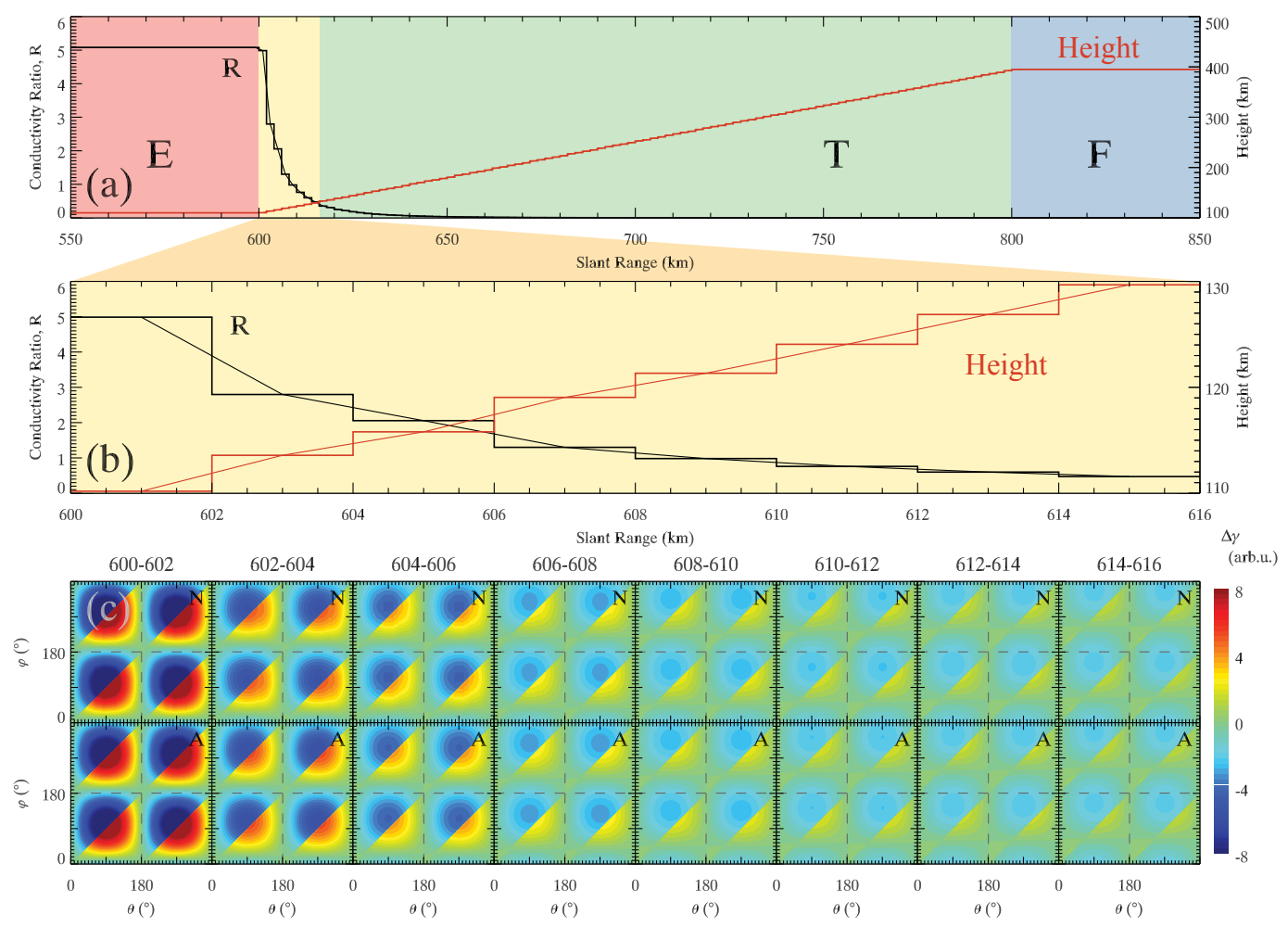

Figure 5. Analysis of the transitional region. (a) The average conductivity ratio $R$ (black line) and the radar virtual height (orange line) vs. the slant range for the entire transitional region and parts of the $\mathrm{E}$ and $\mathrm{F}$ regions. The scales for the conductivity ratio and height are given on the left and right of (a), respectively. The E (red), transitional (yellow and green), and F (blue) regions are highlighted. The region highlighted with yellow is examined more closely in (b) and (c). (b) is similar to (a) but focuses on slant ranges 600-616 km, where most of the change in the conductivity ratio occurs. In addition to the $R$ and height continuous variations, it also gives the average value of $R$ for each $2 \mathrm{~km}$ virtual range gate. The same $2 \mathrm{~km}$ virtual gates are considered in (c), which shows a similar analysis to Fig. 4 . The top row shows the results of the numerical approach $(N)$, and the bottom row shows the analytic approach results $(A)$.

gion up to $130 \mathrm{~km}$ in altitude, these conditions may be satisfied for decameter scales (e.g., Fejer et al., 1984). In the F region, the long-wavelength case refers to significantly larger scales $(\sim 1000 \mathrm{~m})$ below which the fluid theory is not necessarily directly applicable. However, observational studies have found the behavior of decameter-scale irregularities in the $\mathrm{F}$ region to be consistent with many fluid theory predictions, including their propagation with the $\boldsymbol{E} \times \boldsymbol{B}$ drift velocity and stronger waves on the trailing edge of gradient reversals. This leads to a common interpretation that decameterscale waves observed by SuperDARN and kilometer-scale waves in the $\mathrm{F}$ region described by a standard fluid approach are closely related, possibly by a turbulent cascade (e.g., Tsunoda, 1988). Within the above-discussed limits of applicability of the basic equation (Eq. 1) employed in the present modeling, GDI is operational throughout the wide range of ionospheric altitudes spanning both the $\mathrm{E}$ and $\mathrm{F}$ regions. This is because there is always a range of propagation and gradient directions for which growth rates are positive in both the $\mathrm{E}$ and $\mathrm{F}$ regions.
In this study we used the SuperDARN radar at McMurdo Station, Antarctica, to model spatial variations of the GDI growth rate within this radar's field of view and the resulting asymmetries near gradient reversals. Despite this, the results should be also applicable to any of the high-latitude SuperDARN radars in either the Northern or Southern Hemisphere which observe large-scale density structures with gradient reversals. To be more accurate, the parameters of the neutral density model would have to be changed to match a particular location, but these corrections would be very minor. More generally, these results should be the same for any oblique scanning coherent scatter radar at high latitudes. In principle, this technique could also be applied to auroral and mid-latitude radars, but at these latitudes the Earth's magnetic field is further away from the vertical, which affects the assumption that $\boldsymbol{B}$ is perpendicular to the radar's FoV and all other vector quantities are within the same plane. In addition, extended gradient reversals are much rarer at mid- and auroral latitudes than in the polar cap, so this model may be less applicable for interpreting observations in these regions. 
Although this study is relevant to observations with oblique scanning high-frequency (HF) radars, care should be taken with how the results are used to interpret experimental data. In this study, only the linear growth rate of GDI was considered. Even though radar backscatter power and occurrence trends are often interpreted using the GDI growth rates in the linear regime (e.g., Oksavik et al., 2012; Moen et al., 2012), there are other factors that may affect echo power and occurrence. In the polar cap, HF radars rely on beam refraction through a dense ionosphere to observe field-aligned irregularities (Bristow et al., 2011; Koustov et al., 2014). In addition, high background electron density in both the $\mathrm{E}$ and $\mathrm{F}$ regions can smooth gradients required for GDI and limit instability growth (Ruohoniemi and Greenwald, 1997; Koustov et al., 2004). Large conductivity in the E region can also short-out F-region irregularities (Danskin et al., 2002; Kane et al., 2012; Lamarche and Makarevich, 2015). An enhanced D region may act to attenuate backscatter signals, although this effect is not always obvious (Danskin et al., 2002). Nonlinear processes in the F region have been theoretically shown to alter the dependence on the propagation direction while still showing significant anisotropy similar to that predicted by the linear theory (Keskinen and Ossakow, 1981, 1982, 1983). The situation in the E region appears to be somewhat different, with some echoes also exhibiting strong anisotropy (e.g., Ierkic et al., 1980; Makarevich, 2008) and other echoes showing isotropic power variation (Ierkic et al., 1980; Koustov et al., 2001) presumably because of nonlinear processes. The results of the present study are thus more likely to apply in the $\mathrm{F}$ region.

The present model used an idealized gradient reversal superimposed on the radar's FoV, meant to simulate an elongated structure with higher density than the background, such as elongated polar patches (Hosokawa et al., 2014) or Sunaligned arcs (Koustov et al., 2012). In reality, any structure like this will be spread spatially, and the pattern of GDI growth rate around it will be more complicated. In addition, plasma flows around the gradient reversal may also be more complicated than the simple uniform flow considered here. Sun-aligned arcs in particular are known to have complex shears in plasma flow locally across the density enhancement due to converging electric fields (e.g., Kozlovsky et al., 2007), which would complicate growth rate calculations. However, the present model focused on the large-scale motion of density structures, which tends to be relatively consistent on timescales of at least half an hour, and may be still valid for an overall picture of where greater structuring is anticipated.

The results from Fig. 4 show that $\Delta \gamma$ is almost always negative in the $\mathrm{F}$ region, which implies that the growth rate on the trailing edge of a large-scale density structure is almost always larger than that on the leading edge. This agrees with previous observations of polar patches and Sun-aligned arcs in the F-region ionosphere that consistently show a greater irregularity intensity on the trailing edge than the leading edge of these structures (Weber et al., 1984; Milan et al., 2002; Koustov et al., 2012). Greater power from radar backscatter is indicative of more decameter-scale structuring, which agrees with a greater growth rate on the trailing edge. Some studies have shown that radar backscatter (and presumably plasma structuring) occurs within the entire volume of polar patches (Hosokawa et al., 2009). The present model cannot replicate this result as it does not consider structures of arbitrary shapes. The observed patches are often irregularly shaped, which could result in a limited number of places along their edges where the relative gradient/plasma drift orientation is such that no growth would occur (Hosokawa et al., 2009). In this way, these observations are not necessarily inconsistent with the results presented here, but they cannot be represented by the model in its current form.

In the $\mathrm{E}$ region, large values of $\Delta \gamma$ that are both positive and negative indicate that the growth rate can be high on both the leading and trailing edge. Several previous studies have investigated large-scale density structures in the E region (Milan et al., 1999, 2001, 2002), but none of these have focused on asymmetries in irregularity characteristics associated with gradient reversals, so it is difficult to compare these modeling results with observations.

Koustov et al. (2008) presented data in their Fig. 5 that showed smaller echo power on the leading edge of a Sunaligned arc in the $\mathrm{F}$ region but strong backscatter power on both sides of the arc in the short range gates, corresponding to the $\mathrm{E}$ region or transitional region. The arc was approximately parallel to the radar boresight and moved towards the left of the radar FoV, corresponding to $\Delta \gamma \approx 0$ in our model $\left(\theta=90^{\circ}, \phi \approx 180^{\circ}\right.$ in Fig. 4c). Slight changes in the convection direction $(\phi)$ could create both the condition where $\Delta \gamma>0$ (greater growth on leading edge) or $\Delta \gamma<0$ (greater growth on trailing edge). Although this proposed mechanism seems reasonable, it is important to note that the Sun-aligned arc examined in Koustov et al. (2008) has been mapped to an altitude of $250 \mathrm{~km}$, and there is no information about its vertical extent, so it is possible that the density enhancement does not reach the $\mathrm{E}$ region at all and the structuring is due to a different process.

An important new result of this study was that the difference in growth rate depends strongly on the orientation of the gradient reversal and convection direction in both the $\mathrm{E}$ and $\mathrm{F}$ regions. In general, the maximum $\Delta \gamma$ occurred at $\theta=90^{\circ}, 270^{\circ}$ in both the $\mathrm{E}$ and $\mathrm{F}$ regions, regardless of $\phi$. This corresponds to the gradient reversal being aligned along the radar boresight, with the gradient direction being perpendicular to the boresight. On one hand, this results is perhaps not surprising since this is the direction where the peak of the growth rate $\gamma$ itself occurs (Makarevich, 2014). On the other hand, we found that this is also the direction of the peak in the difference $\Delta \gamma$ and in the average difference $\Delta \gamma_{\text {avg }}$, and this latter result in particular does not immediately follow from the growth rate behavior itself. 
In the $\mathrm{F}$ region, the peak in the $\Delta \gamma_{\mathrm{avg}}$ magnitude occurs when the convection direction is perpendicular to the radar boresight $\phi=90^{\circ}$. In the $\mathrm{E}$ region, the peak is when the convection direction is either parallel or antiparallel to the boresight $\phi=0^{\circ}, 180^{\circ}$. This corresponds to the convection direction being parallel to the wave vector. In the transitional region, the "optimal" convection direction changes gradually from $\phi=0^{\circ}$ to $\phi=90^{\circ}$. This is a prediction that has not been tested experimentally. To test it, one would need to follow the track of a large elongated polar patch or Sun-aligned arc. Although polar patches can retain their form in the $\mathrm{F}$ region for several hours (Hosokawa et al., 2014), it is likely that the patch would move a significant lateral distance from the boresight. The structure may have to be observed by multiple SuperDARN radars from different directions unless a highly elongated patch $(>1500 \mathrm{~km})$ is observed, similar to that described in Hosokawa et al. (2014).

Another challenge in observing altitudinal transitions is associated with requirements for a large density structure to have a significant vertical extent, at least from the lower $F$ region through a significant portion of the transitional region. The vertical extent of these structures has not been investigated systematically, although several examples of density enhancements associated with these structures have been presented. Thus Schlatter et al. (2013) examined density enhancements due to high-energy auroral precipitation in the $\mathrm{E}$ and $\mathrm{F}$ region between $\sim 100$ and $\sim 150 \mathrm{~km}$; see their Fig. 1a. Jayachandran et al. (2012) estimated the maximum vertical thickness of Sun-aligned arcs as $\sim 84 \mathrm{~km}$. Sun-aligned arcs have been modeled to have regions of enhanced density at altitudes as low as $100 \mathrm{~km}$ and as high as $300 \mathrm{~km}$ (Crain et al., 1994). This is somewhat supported by observations that show high-density areas at a similar range of altitudes (Valladares and Carlson, 1991). These previous investigations thus provide some support for the idea that it may be possible to find a single density structure with large enough vertical extent to cover the necessary range.

\section{Conclusions}

A model of asymmetries in plasma wave generation due to sharp reversals in the plasma density gradient near elongated density structures in the high-latitude ionosphere revealed the following:
1. Growth rate of plasma irregularities generated in the linear regime of the gradient-drift instability exhibits asymmetry between leading and trailing edges of convecting elongated density structures such as polar patches. Both the GDI growth rate and its asymmetry expressed as the average difference between growth rates on the leading and trailing edges are strongly dependent on the wave propagation direction. Asymmetry is maximized for wave vectors parallel or antiparallel to the elongation direction of the gradient reversal at any altitude in the lower ionosphere.

2. Asymmetry is also strongly dependent on the background convection direction relative to the gradient reversal, but this dependence changes with altitude. Maximum asymmetry occurs when the background convection is parallel to a gradient reversal in the $\mathrm{E}$ region but perpendicular to a gradient reversal in the $\mathrm{F}$ region. There is a relatively smooth transition between configurations associated with maximum asymmetry in the $\mathrm{E}$ and $\mathrm{F}$ layers. In the $\mathrm{E}$ region, either the leading or trailing edge can have significant positive (or negative) growth rate, resulting in the asymmetry favoring either edge, depending on the structure orientation. This is in contrast with the $\mathrm{F}$ region, where growth rate on the trailing edge is almost always larger than the growth rate on the leading edge, albeit with a much lower absolute maximum than in the $\mathrm{E}$ region. This strong altitudinal dependence is due to the rapid decrease of the ratio of Hall and Pedersen conductivities at higher altitudes.

3. An oblique scanning radar is optimally oriented to observe large-scale and elongated density structures in the high-latitude ionosphere when the radar's boresight is closest in direction to the structure elongation for any given altitude and convection direction since this configuration is associated with the largest contrast between leading and trailing edges. The convection direction that leads to the largest contrast depends on the altitude, and for $\mathrm{HF}$ radars that predominantly receive backscatter from the $\mathrm{F}$ region the optimal convection direction is perpendicular to the boresight. 


\section{Appendix A: Analytic expression for the GDI growth rate difference}

In this section, we derive an analytic expression for the average difference in the GDI growth rate across the gradient reversal passing through the radar's viewing area (Fig. 1c). The starting point is Eq. (1). Consider a single point that lies on the gradient reversal within the radar's FoV. In the geometry illustrated in Fig. 1c, the relevant vectors at this point can be written as $\hat{\boldsymbol{k}}=-\sin \alpha \hat{\boldsymbol{e}}_{1}-\cos \alpha \hat{\boldsymbol{e}}_{2}, \hat{\boldsymbol{e}}=-\sin \phi \hat{\boldsymbol{e}}_{1}+\cos \phi \hat{\boldsymbol{e}}_{2}$, and $\hat{\boldsymbol{g}}= \pm\left[\sin \theta \hat{\boldsymbol{e}}_{1}-\cos \theta \hat{\boldsymbol{e}}_{2}\right]$, where angles $\theta$ and $\phi$ were previously defined in Sect. 2, angle $\alpha$ is the angle between the wave vector and the boresight direction, and unit vectors $\hat{\boldsymbol{e}}_{1}$ and $\hat{\boldsymbol{e}}_{2}$ are perpendicular and parallel to the boresight, respectively.

Using these expressions, all the terms in Eq. (1) can be expressed as functions of $\alpha, \theta$, and $\phi$ :

$\gamma= \pm \sin (\alpha+\theta)[\cos (\alpha+\phi)-R \sin (\alpha+\phi)]$.

This is the GDI growth rate at a given point for the two opposing gradient directions that will occur on either side of the gradient reversal. If $\phi<\theta-180^{\circ}$ or $\theta<\phi<\theta+180^{\circ}$, the negative sign represents the leading edge and the positive sign represents the trailing edge, so the growth rate difference is negative. If $\phi<\theta$ or $\theta+180^{\circ}<\phi<\theta+360^{\circ}$, the positive sign represents the leading edge and the negative sign the trailing edge, so the growth rate difference is positive. The expression for growth rate difference is then given by

$\Delta \gamma=\left\{\begin{array}{c}2 \sin (\alpha+\theta)[\cos (\alpha+\phi)-R \sin (\alpha+\phi)], \\ \phi<\theta \text { or } \theta+180^{\circ}<\phi<\theta+360^{\circ}, \\ -2 \sin (\alpha+\theta)[\cos (\alpha+\phi)-R \sin (\alpha+\phi)], \\ \phi<\theta-180^{\circ} \text { or } \theta<\phi<\theta+180^{\circ} .\end{array}\right.$

Equation (A2) can be expressed as one equation using the Heaviside step function $\Theta$. First, let us define two functions of $\theta$ and $\phi$ :

$H_{1}(\theta, \phi) \equiv \Theta((\theta-\pi)-\phi)+\Theta(\phi-\theta)-\Theta(\phi-(\theta+\pi))$,

$H_{2}(\theta, \phi) \equiv \Theta(\phi-(\theta-\pi))-\Theta(\phi-\theta)+\Theta(\phi-(\theta+\pi))$.
Equation (A3) gives $H_{1}(\theta, \phi)$, which returns 1 for $\phi<\theta-$ $180^{\circ}$ and $\theta<\phi<\theta+180^{\circ}$, and 0 for all other values of $\phi$. Equation (A4) gives $H_{2}(\theta, \phi)$, which returns 1 for $\phi<\theta$ and $\theta+180^{\circ}<\phi<\theta+360^{\circ}$, and 0 for all other values of $\phi$. A single expression for $\Delta \gamma$ is constructed using these two function as

$$
\Delta \gamma=2 H_{1}(\theta, \phi) \sin (\alpha+\theta)[\cos (\alpha+\phi)-R \sin (\alpha+\phi)]
$$

$-2 H_{2}(\theta, \phi) \sin (\alpha+\theta)[\cos (\alpha+\phi)-R \sin (\alpha+\phi)]$.

This expression can be rewritten in a shorter form if we define $H(\theta, \phi) \equiv H_{2}(\theta, \phi)-H_{1}(\theta, \phi)$ :

$\Delta \gamma=-2 H(\theta, \phi) \sin (\alpha+\theta)[\cos (\alpha+\phi)-R \sin (\alpha+\phi)]$.

Equation (A6) gives the difference in the GDI growth rate across the gradient reversal for any point along the reversal for any arbitrary orientation of the gradient reversal and convection direction. To find the average difference in growth rate, we integrate over $\alpha$ across the radar FoV between $-\alpha_{0}$ and $+\alpha_{0}$ with respect to the boresight. In this integration, we assume that the conductivity ratio $R$ is a constant for any combination of angles, which is a fair assumption for observations in the $\mathrm{E}$ region or in the $\mathrm{F}$ region.

$$
\begin{aligned}
\Delta \gamma_{\text {avg }}= & \frac{1}{2 \alpha_{0}} \int_{-\alpha_{0}}^{\alpha_{0}} \Delta \gamma \mathrm{d} \alpha=-\frac{H(\theta, \phi)}{\alpha_{0}} \int_{-\alpha_{0}}^{\alpha_{0}} \\
& \sin (\alpha+\theta)[\cos (\alpha+\phi)-R \sin (\alpha+\phi)] \mathrm{d} \alpha
\end{aligned}
$$

The integral in Eq. (A7) is easily evaluated, and the final expression for the average difference in GDI growth rate across the gradient reversal becomes

$$
\begin{aligned}
\Delta \gamma_{\mathrm{avg}}= & H(\theta, \phi)[\sin (\phi-\theta)-C \sin (\phi+\theta) \\
& +R(\cos (\phi-\theta)-C \cos (\phi+\theta))],
\end{aligned}
$$

where a new constant has been defined: $C \equiv \sin \left(2 \alpha_{0}\right) / 2 \alpha_{0}$. For a typical SuperDARN radar, $\alpha_{0}=26^{\circ}$ and $C \approx 0.87$. 
Acknowledgements. This work was supported by NSF grants AGS1248127 and PLR-1443504. The MSISE-90 model is available from the GSFC/SPDF OMNIWeb interface at http://omniweb.gsfc. nasa.gov/.

The topical editor, S. Milan, thanks two anonymous referees for help in evaluating this paper.

\section{References}

Bristow, W. A., Spaleta, J., and Parris, R. T.: First observations of ionospheric irregularities and flows over the south geomagnetic pole from the Super Dual Auroral Radar Network (SuperDARN) HF radar at McMurdo Station, Antarctica, J. Geophys. Res., 116, A12325, doi:10.1029/2011JA016834, 2011.

Chisham, G., Lester, M., Milan, S. E., Freeman, M. P., Bristow, W. A., Grocott, A., McWilliams, K. A., Ruohoniemi, J. M., Yeoman, T., Dyson, P. L., Greenwald, R. A., Kikuchi, T., Pinnock, M., Rash, J. P. S., Sato, N., Sofko, G. J., Villain, J.P., and Walker, A. D. M.: A decade of the Super Dual Auroral Radar Network (SuperDARN): scientific achievements, new techniques and future directions, Surv. Geophys., 28, 33-109, doi:10.1007/s10712-007-9017-8, 2007.

Chisham, G., Yeoman, T. K., and Sofko, G. J.: Mapping ionospheric backscatter measured by the SuperDARN HF radars - Part 1: A new empirical virtual height model, Ann. Geophys., 26, 823841, doi:10.5194/angeo-26-823-2008, 2008.

Crain, D. J., Sojka, R. W., and Zhu, L.: Modeling Sun-aligned polar cap arcs, Radio Sci., 29, 269-281, 1994.

Danskin, D. W., Koustov, A. V., Ogawa, T., Nishitani, N., Nozawa, S., Milan, S. E., Lester, M., and Andre, D.: On the factors controlling occurrence of F-region coherent echoes, Ann. Geophys., 20, 1385-1397, doi:10.5194/angeo-20-1385-2002, 2002.

Fejer, B.-G., Providakes, J., and Farley, D.-T.: Theory of Plasma Waves in the Auroral E Region, J. Geophys. Res., 89, 74877494, 1984.

Forsythe, V. V. and Makarevich, R. A.: Dual radar investigation of E region plasma waves in the southern polar cap, J. Geophys. Res., 120, 9132-9147, doi:10.1002/2015JA021664, 2015.

Hedin, A. E.: Extension of the MSIS thermospheric model into the middle and lower Atmosphere, J. Geophys. Res., 96, 1159-1172, 1991.

Hosokawa, K., Shiokawa, K., Otsuka, Y., Ogawa, T., St-Maurice, J.-P., Sofko, G. J., and Andre, D. A.: Relationship between polar cap patches and field-aligned irregularities as observed with and all-sky airglow imager at Resolute Bay and the PolarDARN radar at Rankin Inlet, J. Geophys. Res., 114, A03306, doi:10.1029/2008JA013707, 2009.

Hosokawa, K., Taguchi, S., Shiokawa, K., Otsuka, Y., Ogawa, Y., and Nicolls, M.: Global imaging of polar cap patches with dual airglow imagers, Geophys. Res. Lett., 41, 1-6, doi:10.1002/2013GL058748, 2014.

Ierkic, H. M., Fejer, B. J., and Farley, D. T.: The dependence on zenith angle of the strength of 3-meter equatorial electrojet irregularities, Geophys. Res. Lett., 7, 497-500, 1980.

Jayachandran, P. T., Hosokawa, K., Shiokawa, K., Otsuka, Y., Watson, C., Mushini, S. C., MacDougall, J. W., Prikryl, P., Chadwick, R., and Kelly, T. D.: GPS total electron content variations associated with poleward moving Sun-aligned arcs, J. Geophys. Res., 117, A05310, doi:10.1029/2011JA017423, 2012.

Kane, T. A., Makarevich, R. A., and Devlin, J. C.: HF radar observations of ionospheric backscatter during geomagnetically quiet periods, Ann. Geophys., 30, 221-233, doi:10.5194/angeo-30221-2012, 2012.

Keskinen, M. J. and Ossakow, S. L.: On the spatial power spectrum of the $\boldsymbol{E} \times \boldsymbol{B}$ gradient drift instability in ionospheric plasma clouds, J. Geophys. Res., 86, 6947-6950, 1981.

Keskinen, M. J. and Ossakow, S. L.: Nonlinear evolution of plasma enhancements in the auroral ionosphere. I - Long wavelength irregularities, J. Geophys. Res., 87, 144-150, 1982.

Keskinen, M. J. and Ossakow, S. L.: Theories of high-latitude ionospheric irregularities: A review, Radio Sci., 18, 1077-1091, 1983.

Koustov, A., Nishitani, N., Ebihara, Y., Kikuchi, T., Hairston, M. R., and Andre, D.: Subauroral polarization streams: observations with the Hokkaido and King Salmon SuperDARN radars and modeling, Ann. Geophys., 26, 3317-3327, doi:10.5194/angeo26-3317-2008, 2008.

Koustov, A. V., Igarashi, K., André, D., Ohtaka, K., Sato, N., Yamagishi, H., and Yukimatu, A.: Observations of 50- and $12-\mathrm{MHz}$ auroral coherent echoes at the Antarctic Syowa station, J. Geophys. Res., 106, 12875-12887, 2001.

Koustov, A. V., Sofko, G. J., André, D., Danskin, D. W., and Benkevitch, L. V.: Seasonal variation of HF radar F region echo occurrence in the midnight sector, J. Geophys. Res., 109, A06305, doi:10.1029/2003JA010337, 2004.

Koustov, A. V., Hosokawa, K., Nishitani, N., Shiokawa, K., and Liu, H.: Signatures of moving polar cap arcs in the F-region PolarDARN echoes, Ann. Geophys., 30, 441-455, doi:10.5194/angeo30-441-2012, 2012.

Koustov, A. V., Ponomarenko, P. V., Ghezelbash, M., Themens, D. R., and Jayachandran, P. T.: Electron density and electric field over Resolute Bay and $\mathrm{F}$ region ionospheric echo detection with the Rankin Inlet and Inuvik SuperDARN radars, Radio Sci., 49, 1194-1205, doi:10.1002/2014RS005579, 2014.

Kozlovsky, A., Aikio, A., Turunen, T., Nilsson, H., Sergienko, T., Safargaleev, V., and Kauristie, K.: Dynamics and electrical currents of morningside sun-aligned auroral arcs, J. Geophys. Res., 112, A06306, doi:10.1029/2006JA012244, 2007.

Lamarche, L. J. and Makarevich, R. A.: Solar control of F region radar backscatter: Further insights from observations in the southern polar cap, J. Geophys. Res., 120, 9875-9890, doi:10.1002/2015JA021663, 2015.

Makarevich, R. A.: HF radar observations of high-velocity E-region echoes from the eastward auroral electrojet, J. Geophys. Res., 113, A09321, doi:10.1029/2008JA013204, 2008.

Makarevich, R. A.: Symmetry considerations in the two-fluid theory of the gradient-drift instability in the lower ionosphere, J. Geophys. Res., 119, 7902-7913, doi:10.1002/2014JA020292, 2014.

Makarevitch, R. A., Honary, F., McCrea, I. W., and Howells, V. S. C.: Imaging riometer observations of drifting absorption patches in the morning sector, Ann. Geophys., 22, 3461-3478, doi:10.5194/angeo-22-3461-2004, 2004.

Makarevich, R. A., Lamarche, L. J., and Nicolls, M. J.: Resolute Bay Incoherent Scatter Radar observations of plasma structures in the vicinity of polar holes, J. Geophys. Res., 120, 7970-7986, doi:10.1002/2015JA021443, 2015. 
Milan, S. E., Davies, J. A., and Lester, M.: Coherent HF radar backscatter characteristics associated with auroral forms identified by incoherent radar techniques: A comparison of CUTLASS and EISCAT observations, J. Geophys. Res., 104, 22591-22604, 1999.

Milan, S. E., Lester, M., Sato, N., and Takizawa, H.: On the altitude dependence of the spectral characteristics of decametrewavelength $\mathrm{E}$ region backscatter and the relationship with optical auroral forms, Ann. Geophys., 19, 205-217, doi:10.5194/angeo19-205-2001, 2001.

Milan, S. E., Sato, N., Lester, M., Yeoman, T. K., Murata, Y., Doi, $\mathrm{H}$., and Saemundsson, T.: The spectral characteristics of E-region radar echoes co-located with and adjacent to visual auroral arcs, Ann. Geophys., 20, 795-805, doi:10.5194/angeo-20-795-2002, 2002.

Moen, J., Oksavik, K., Abe, T., Lester, M., Saito, Y., Bekkeng, T. A., and Jacobsen, K. S.: First in situ measurements of HF radar echoing targets, Geophys. Res. Lett., 39, L07104, doi:10.1029/2012GL051407, 2012.

Oksavik, K., Moen, J., Lester, M., Bekkeng, T. A., and Bekkeng, J. K.: In situ measurements of plasma irregularity growth in the cusp ionosphere, J. Geophys. Res., 117, A11301, doi:10.1029/2012JA017835, 2012.
Ruohoniemi, J. M. and Greenwald, R. A.: Rates of scattering occurrence in routine HF radar observations during solar cycle maximum, Radio Sci., 32, 1051-1070, 1997.

Schlatter, N. M., Ivchenko, N., Sergienko, T., Gustavsson, B., and Brändström, B. U. E.: Enhanced EISCAT UHF backscatter during high-energy auroral electron precipitation, Ann. Geophys., 31, 1681-1687, doi:10.5194/angeo-31-1681-2013, 2013.

Schunk, R. W. and Nagy, A. F.: Electron temperatures in the F region of the ionosphere: Theory and observations, Rev. Geophys. Space Ge., 16, 355-399, 1978.

Tsunoda, R. T.: High-latitude F region irregularities - A review and synthesis, Geophys. Rev., 26, 719-760, 1988.

Valladares, C.-E. and Carlson, H.-C.: The electrodynamic, thermal, and energetic character of intense Sun-aligned arcs in the polar cap, J. Geophys. Res., 96, 1379-1400, 1991.

Weber, E. J., Buchau, J., Moore, J. G., Sharber, J. R., Livingston, R. C., Winningham, J. D., and Reinisch, B. W.: F layer ionization patches in the polar cap, J. Geophys. Res., 89, 1683-1694, 1984. 